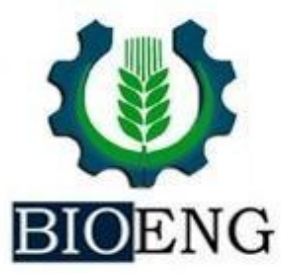

\title{
APLICAÇÃO DO MODELO PIRFICT NO BANCO DE DADOS DA RIMAS
}

\author{
G. L. Barbosa ${ }^{1 *}$, R. Kirchheim ${ }^{3}$, G. N. Santos ${ }^{3}$, R. L. \\ Manzione $^{1}$; W. A. L. Zanetti ${ }^{2}$
}

\begin{abstract}
${ }^{1}$ UNESP - Universidade Estadual Paulista, Faculdade de Ciências e Engenharia (FCE), Tupã, SP, Brasil ${ }^{2}$ UNESP - Universidade Estadual Paulista, Faculdade de Ciências Agronômicas (FCA), Botucatu, SP, Brasil

${ }^{3}$ CPRM - Serviço Geológico do Brasil, Superintendência de São Paulo, São Paulo - SP, Brasil
\end{abstract}

Article history: Received 10 March 2020; Received in revised form 21 April 2020; Accepted 21 April 2020; Available online 30 September 2020.

\section{RESUMO}

A água é considerada um recurso finito de grande importância para manutenção e subsistência dos seres vivos. O continuo crescimento das demandas torna necessário o desenvolvimento e planejamento de técnicas para supervisão e gestão dos recursos hídricos. Uma considerável parcela disponível de água doce para consumo encontra-se na forma de águas subterrâneas e que precisam ser monitoradas e usadas de forma racional, com o intuito de evitar usos não sustentáveis e explorações descontroladas. Para tanto, existem redes de monitoramento de água subterrâneas, tal qual a Rede Integrada de Monitoramento de Águas Subterrâneas (RIMAS), operada e mantida pelo Serviço Geológico do Brasil (CPRM). O presente estudo versa sobre a aplicação do software Menyanthes neste conjunto de dados como uma forma de atribuir-lhes consistência estatística e lograr preencher lacunas na referida série observada. $O$ referido programa consiste na modelagem de causa e efeito, relacionando o histórico de chuvas com os respectivos piezogramas. Os resultados obtidos por intermédio desta aplicação fornecem perspectivas de normatizar a sistemática de preenchimento de lacunas na rede RIMAS, assim como também caminhos para prognósticos de comportamento freático frente a eventos climáticos como estiagens, similares à ocorrida entre os anos de 2013 e 2015 na região sudeste do Brasil.

Palavras-chave: águas subterrâneas; banco de dados; níveis freáticos; software Menyanthes.

\section{APPLICATION OF THE PIRFICT MODEL IN THE RIMAS DATABASE}

\begin{abstract}
Water is considered a finite resource of great importance for the maintenance and subsistence of living beings. The continuous growth of water demands makes it necessary to develop management strategies for the water resources use and protection. The groundwater resources builds up a considerable portion of the fresh water overall availability and need to be consumed rationally and in a sustainable way. Therefore, groundwater monitoring networks, such as the Integrated Groundwater Monitoring
\end{abstract}

*glbarbosa3105@gmail.com 
Network (RIMAS), operated and maintained by the Geological Survey of Brazil (CPRM), are fundamental strategies. The present study deals with the application of the Menyanthes Software as a way of attributing statistical consistency to this data bank and to fill missing data in the observed series. The main rationale is to build up a model relating the rainfall background to the respective observed groundwater levels. The results obtained through this application provide standardized approaches for consistency in the RIMAS data bank, as well as tools for prognosis for the phreatic behavior amidst climatic events such as droughts, similar to what occurred between the years 2013 and 2015 in the southeast region of Brazil.

Keywords: groundwater; database; phreatic levels; Menyanthes Software.

\section{INTRODUÇÃO}

A água é fundamental na manutenção e sobrevivência da vida, recurso básico para o desenvolvimento das mais diversas atividades humanas, entre elas, geração de energia, agricultura, indústria, transporte, turismo, lazer, entre outros. Esta condição vem impulsionando as sociedades a desenvolver técnicas para maximizar o aproveitamento deste recurso.

No entanto, ainda que o planeta apresente $3 / 4$ da superfície coberta por água, apenas uma pequena parcela da mesma encontra-se disponível para consumo. As águas subterrâneas armazenadas nos aquíferos representam a parcela mais significativa desta parcela disponível para uso. Este fato traz à tona a importância da proteção e uso sustentável das mesmas, tanto do ponto de vista de quantidade como de qualidade.

A exploração descontrolada e a falta de políticas públicas apropriadas afetam sobremaneira a manutenção de suas qualidades e funções. Reside neste fato a importância de contar com estudos e estratégias de gestão sustentável. Trata-se da única forma de garantir acesso a este recurso às gerações atuais e futuras (Kirchheim et al., 2018)

Redes de monitoramento de água subterrânea constituem-se em ferramentas indispensáveis para a gestão das águas subterrâneas. A referência no âmbito do Brasil é a Rede Integrada de Monitoramento de Águas Subterrâneas - RIMAS, operada e mantida pelo Serviço Geológico do Brasil (CPRM). Esta sistemática de monitoramento permite $\mathrm{O}$ desenvolvimento de balanços hídricos, estimações de recargas e a construção e calibração de modelos matemáticos para fins de gestão.

Neste contexto, este trabalho tem como objetivo a aplicação do programa Menyanthes (KWR, 2012) ao banco de dados de níveis freáticos fornecidos pelo RIMAS. O referido programa consiste na modelagem de causa e efeito, relacionando o histórico de chuvas com os respectivos piezogramas. Os resultados obtidos por intermédio desta aplicação fornecem perspectivas de normatizar a sistemática de preenchimento de lacunas na rede RIMAS, assim como também caminhos para prognósticos de comportamento freático frente a eventos climáticos como estiagens, similares à ocorrida entre os anos de 2013 e 2015 na região sudeste do Brasil.

\section{ÁREA DE ESTUDO}

Este estudo foi desenvolvido na região Sudeste do Brasil, em poços de monitoramento da rede RIMAS nos Sistemas Aquíferos Bauru (SAB) e Guarani (SAG). Segundo Santos et al., 2018, a rede RIMAS é constituída por poços piezométricos (doravante PM's) 
implantados e operados pelo Serviço Geológico do Brasil - CPRM nos principais aquíferos livres e porosos brasileiros. Seu objetivo é acompanhar as alterações espaciais e temporais na qualidade e quantidade das águas subterrâneas desses aquíferos como insumo para a gestão integrada dos recursos hídricos e dar suporte às estratégias e políticas de uso, proteção e conservação dos recursos hídricos subterrâneos. O monitoramento quantitativo é feito através de medidores de nível automáticos (level loggers), para medição (horária) do nível estático e plataformas automáticas de coleta de dados (PCD's), para aquisição dos dados pluviométricos a cada $15 \mathrm{~min}$. Já o monitoramento qualitativo é realizado anualmente através da análise dos parâmetros químicos e físico-químicos estabelecidos pela resolução CONAMA 396/2008. Dentre o conjunto de PM's, foram selecionados poços de monitoramento representativos, porém com presença de lacunas nos registros. O Quadro 1 fornece as informações básicas dos PM's analisados.

Quadro 1. Localidade dos poços analisados, junto com informações básicas dos PM's.

\begin{tabular}{cccccc}
\hline Município & UF & Lat. & Log. & Aflo. & Prof. \\
\hline Ivinhema & MS & 22,36 & 53,91 & SAB & 66 \\
Aparecida do Taboado & MS & 20,14 & 51,14 & SAB & 52 \\
Camapuã & MS & 19,63 & 54,08 & SAB & 62 \\
Coxim & MS & 18,47 & 54,73 & SAB & 21,6 \\
Iguatemi & MS & 23,66 & 54,56 & SAB & 62 \\
Nioaque & MS & 21,16 & 55,81 & SAG & 50 \\
Rochedo & MS & 19,98 & 54,78 & SAG & 66 \\
São Gabriel do Oeste & MS & 9,40 & 54,69 & SAG & 60 \\
Santa Rita do pardo & MS & 21,30 & 52,81 & SAB & 60 \\
Inocência & MS & 19,73 & 51,93 & SAB & 66 \\
Figueirão & MS & 18,68 & 53,63 & SAG & 61,4 \\
Nova Andradina & MS & 22,07 & 53,36 & SAG & 61,4 \\
Porto rico & PR & 22,78 & 53,26 & SAB & 60 \\
União da vitória & PR & 26,19 & 51,09 & SAG & 16,85 \\
Planaltina do Paraná & PR & 23,00 & 52,93 & SAB & 70 \\
Pindorama & SP & 21,22 & 48,90 & SAB & 60 \\
Monte aprazível & SP & 20,77 & 49,73 & SAB & 52 \\
Valparaiso & SP & 21,31 & 50,92 & SAB & 52 \\
Assis & SP & 22,60 & 50,37 & SAB & 60 \\
São Simão & SP & 21,45 & 47,58 & SAG & 46 \\
Brotas & SP & 22,30 & 48,10 & SAG & 33 \\
Bastos & SP & 21,95 & 50,67 & SAB & 52,7 \\
Boa esperança do sul & SP & 21,96 & 48,39 & SAG & 16,4 \\
Meridiano & SP & 20,35 & 50,16 & SAB & 56,64 \\
\hline & & & & &
\end{tabular}

Abaixo a Figura 1 indica a localização geográfica dos PM's avaliados e a distribuição do arcabouço geológico aflorante. 


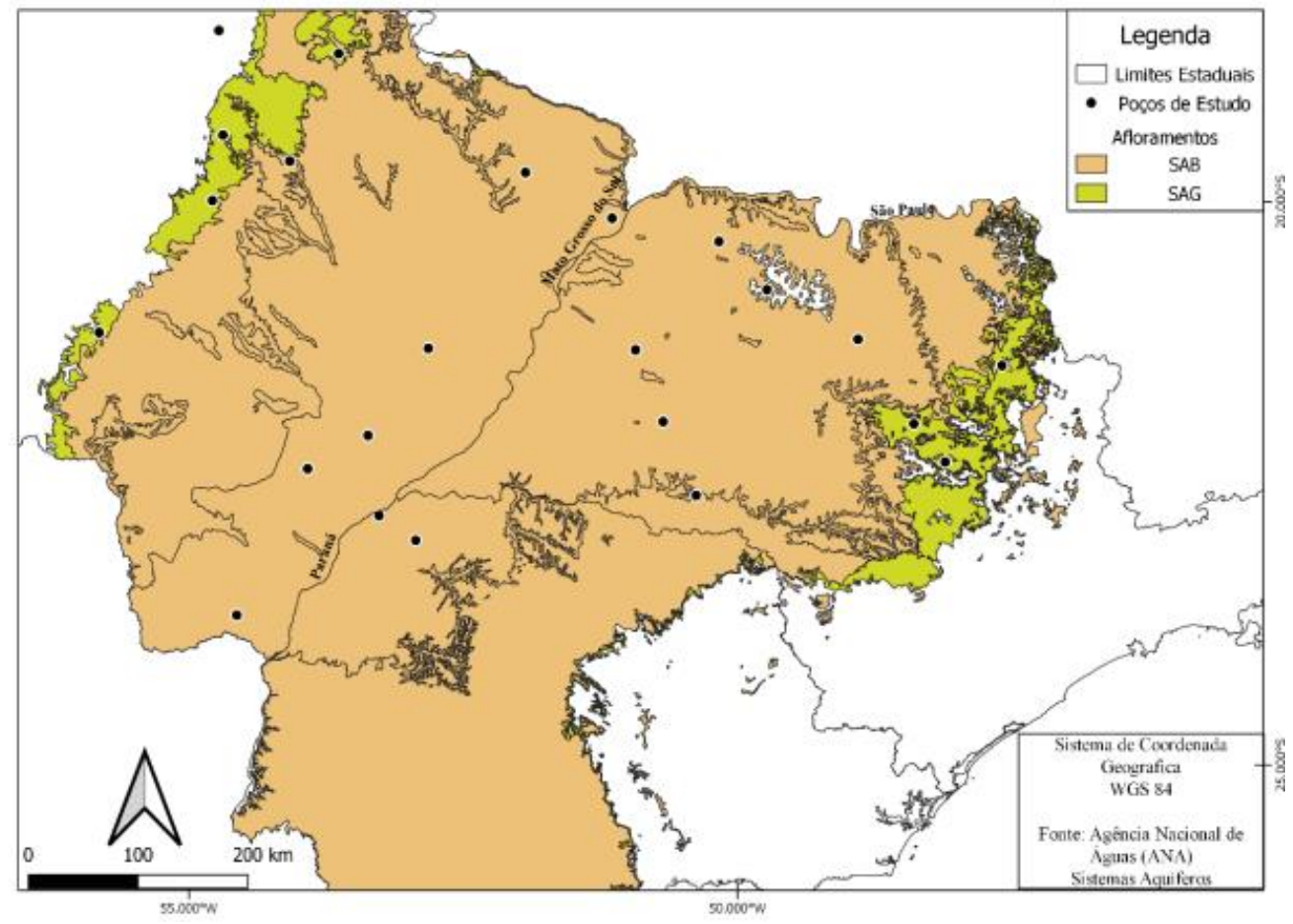

Figura 1. Localidade dos poços junto com a distribuição do arcabouço geológico aflorante

\section{MATERIAIS E MÉTODOS}

A aquisição e monitoramento de dados de nível de água subterranea são de importância fundamental para equilibrar interesses econômicos e ambientais relacionados ao uso de recursos hídricos e do solo (VON ASMUTH; KNOTTERS, 2004). A dinamica da água subterrânea, principalmente sua oscilação de níveis, é explicada na forma de séries temporais. A aplicação do software Menyanthes (KWR, 2012) nos dados gerados pela rede RIMAS permite o desenvolvimento de modelos que reproduzam o comportamento freático atribuindo-lhes significância estatística. Para isso o software utiliza o modelo PIRFICT (Predefined Impulse Response Function, Von Asmuth et al. (2002). Este algoritmo relaciona as séries temporais de nível freático com as séries temporais de precipitação, reduzindo o número de parâmetros e minimizando os problemas relacionados com frequência temporal dos dados (VON ASMUTH et al., 2002; VON ASMUTH; KNOTTERS, 2004, (BOX e JENKINS, 1976; TANKERSLEY e GRAHAM, 1994; VAN GEER; ZUUR, 1997, YI; LEE, 2003). VON ASMUTH et al. (2002) utilizou esta metodologia em avaliações do funcionamento de diques na Holanda. Manzione (2007) destaca a potencialidade de uso para fins de gestão de recursos hídricos subterrâneos com adequabilidade para diferentes sistemas hidrológicos. $\mathrm{O}$ modelo PIRFICT se destaca de acordo com MANZIONE (2010), devido a sua capacidade de lidar com frequência heterogênea de dados de entrada. O método foi projetado para responder a diversos tipos de estresses e, com isso, permitir a manipulação de grandes 
volumes de dados de entrada. Barbosa (2019) utilizou o modelo PIRFICT para avaliar a relação entre os diferentes tipos de utilização de solo e os níveis freáticos monitorados com periodicidade mensal na região de Angatuba-SP.

\section{O MODELO PIRFICT}

O modelo PIRFICT foi desenvolvido para lidar com séries de entrada e saída que possuem diferentes frequências de observações e intervalos de tempo irregulares. Neste modelo os pulsos dos dados de entrada são convertidos em um tempo contínuo que representa de forma alternativa os modelos de Função de Transferência de Ruído (FTR), que possuem aspectos auxiliares na descrição do que ocorre quando os níveis freáticos sofrem interferência de variáveis naturais, como por exemplo, a precipitação.
Considerando um sistema linear, pressupõe-se que, de acordo com as variáveis utilizadas, a série dos níveis freáticos é a variação de uma série de precipitação excedente, com a subtração da evapotranspiração potencial, sendo diretamente relacionada pela função de impulso resposta (IR). A adoção da premissa de que não ocorra nenhum tipo de interferência externa ou perturbação, sendo o sistema influenciado apenas pela precipitação excedente, a abordagem é do tipo linear. Neste sentido o bloco TFN do modelo representa uma convolução integral de operador linear que, a partir de duas funções, fornece uma terceira que relaciona a interação das alturas do lençol freático e a precipitação excedente (VON ASMUTH et al., 2002):

$$
\begin{aligned}
& h(t)=h(t)+d+r(t) \\
& h^{*}(t)=\int_{-\infty}^{t} p(\tau) \theta(t-\tau) \partial \tau \\
& r(t)=\int_{-\infty}^{t} \varnothing(t-\tau) \partial W(\tau)
\end{aligned}
$$

No qual temos:

$h(t)$ é a altura de lençol freático observada no tempo $\mathrm{t}[\mathrm{T}]$;

$h^{*}(t)$ é a altura de lençol freático predita no tempo $t$ creditado ao excedente de precipitação relativa a $d$ [L];

$d$ é o nível de $h^{*}(t)$ sem a precipitação, ou em outras palavras o nível da drenagem local, relativo a superfície do solo [L];

$r(t)$ é a série dos resíduos [L];

$p(\tau)$ é a intensidade do excedente de precipitação no tempo $t[\mathrm{~L} / \mathrm{T}]$; $\theta(t)$ é a função de transferência de impulso/resposta (IR) [-];

$\emptyset(t)$ é a função IR do ruído [-];

$W(\tau)$ é um processo de ruído branco contínuo (Wiener) [L], com propriedades $\quad \mathrm{E}\{\partial W(\tau)\}=0$, $\mathrm{E}[\{\partial W(\tau)\} 2]=\partial(\tau)$, $\mathrm{E}[\partial W(\tau 1) \partial W(\tau 2)]=0, \tau 1 \neq \tau 2$.

Com isso podemos definir o nível da drenagem local d, que é obtido a partir dos dados conforme a Equação (4), sendo $\mathrm{N}$ o número de observações de alturas de lençol freático: 


$$
d=\frac{\sum_{i=0}^{N} h\left(t_{i}\right)}{N}-\frac{\sum_{i=0}^{N} h^{*}\left(t_{i}\right)}{N}-\frac{\sum_{i=0}^{N} r\left(t_{i}\right)}{N}
$$

As condições hidrológicas da área afetam de maneira direta a região e a forma da função IR (Pearson tipo III) de acordo com MANZIONE et al. (2010). A seleção por esse tipo de função se dá por sua natureza flexível, ajustando-se a uma grande gama de respostas hidrológicas. $\quad \mathrm{Se}$

$$
\begin{aligned}
& \theta(t)=A \frac{A^{N} T^{n-1} e^{-a t}}{\Gamma(n)} \\
& \varnothing(t)=\sqrt{2 * \alpha \sigma_{r}^{2}} e^{-a t}
\end{aligned}
$$

A função de distribuição de Pearson III, ou representada pela letra grega $\theta(t)$, assume formas que variam gradualmente desde uma curva considerarmos um sistema linear, a parte determinística da dinâmica do nível freático é integralmente descrita pela função IR. Analisando este caso, os parâmetros podem ser determinados de acordo com VON ASMUTH et al. (2002):

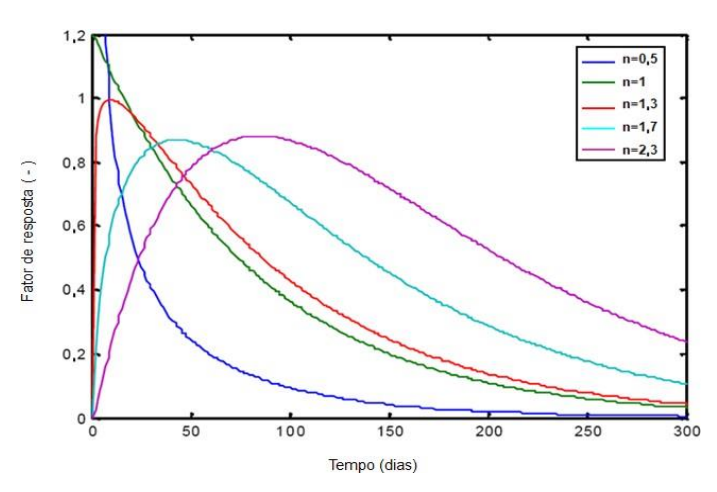

Figura 2. Exemplo do alcance das formas que uma função de distribuição Pearson tipo III. (FONTE: MANZIONE, 2015)

Onde $A, a, n$, são os parâmetros da curva ajustada, $\Gamma(n)$ é a função Gamma, $\alpha$ controla a taxa de decaimento de $\varnothing(t)$ e $\sigma_{r}^{2}$ é a variância dos resíduos. Pode-se então demonstrar as relações entre as variáveis propostas. $\mathrm{O}$ parâmetro A se relaciona com a resistência à drenagem ( $\mathrm{a}$ área da função de IR é igual à razão entre a altura média do lençol freático e a recarga média). O parâmetro $a$ é determinado exponencial a gaussiana, de acordo com as variáveis, como verificado na Figura 2 (MANZIONE, 2010). 
igual a um simples modelo físico de coluna de solo unidimensional, descartando fluxo lateral e o funcionamento da zona não-saturada (KNOTTERS; BIERKENS, 2000).

Em virtude do que foi mencionado, foi utilizado o software
Menyanthes (KWR, 2012), para aplicar o modelo de transferência de ruído PIRFICT na compreensão da relação entre as variáveis climáticas e os níveis dos lençóis freáticos analisados nos poços do RIMAS.

\section{RESULTADOS E DISCUSSÕES}

A aplicação do modelo se baseou na sistematização dos parâmetros principais, a saber, dados de precipitação e de níveis freáticos e possibilitou a caracterização de cada poço de forma individual.

Quadro 2. Parâmetros dos modelos ajustados para os poços em relação à precipitação dos poços localizados no Estado do Paraná.

\begin{tabular}{cccccc}
\hline Poços & NMLF $(\mathrm{m})$ & EVP $(\%)$ & RMSE $(\mathrm{m})$ & UF & Aflo. \\
\hline Assis & -29.057 & 89.89 & 0.363 & SP & SAB \\
Bastos & -5.497 & 55.20 & 0.672 & SP & SAB \\
Boa Esperança do Sul & -4.552 & 41.00 & 0.364 & SP & SAG \\
Brotas & -20.624 & 38.70 & 0.912 & SP & SAG \\
Meridiano & -28.859 & 74.90 & 0.453 & SP & SAB \\
Monte Aprazível & -22.222 & 74.20 & 0.453 & SP & SAB \\
Penápolis & -5.651 & 79.60 & 0.262 & SP & SAB \\
Pindorama & -28.441 & 78.60 & 0.290 & SP & SAB \\
São Simão & -10.690 & 33.40 & 0.685 & SP & SAG \\
Valparaíso & -9.294 & 64.50 & 0.685 & SP & SAB \\
Planaltina do Paraná & -35.676 & 73.70 & 0.751 & PR & SAB \\
Porto Rico & -5.954 & 68.85 & 0.507 & PR & SAB \\
União da Vitória & -3.778 & 49.30 & 0.446 & PR & SAG \\
Aparecida do Tabuado & -15.07 & 91.78 & 0.403 & MS & SAB \\
Camapuã & -35.28 & 50.30 & 0.459 & MS & SAB \\
Coxim & -10.40 & 88.7 & 0.401 & MS & SAB \\
Figueirão & -11.79 & 79.44 & 0.287 & MS & SAG \\
Iguatemi & -3.39 & 79.41 & 0.343 & MS & SAB \\
Inocência & -15.21 & 65.87 & 0.198 & MS & SAB \\
Ivinhema & -38.65 & 76.96 & 0.572 & MS & SAB \\
Nioaque & -5.86 & 92.14 & 0.204 & MS & SAG \\
Nova Andradina & -8.18 & 94.08 & 0.172 & MS & SAG \\
Rochedo & -32.54 & 93.80 & 0.121 & MS & SAG \\
Santa Rita do Rio Pardo & -14.249 & 94.20 & 0.316 & MS & SAB \\
São Gabriel do Oeste & -22.85 & 84.07 & 0.316 & MS & SAG \\
\hline & & & & &
\end{tabular}

O Quadro 2 identifica os resultados obtidos do modelo para os parâmetros estáticos dos poços analisados. (nível médio do lençol freático - NMLF; percentual de variância explicada pelo modelo - EVP; raiz do erro quadrático médio - RMSE).
Verificando a métrica utilizada para designar a relação entre os parâmetros de precipitação e níveis freáticos, nota-se que todos os dados tiveram valores consistentes, mostrando dessa forma, como o modelo se ajusta a diferentes tipos de clima e região. Os 
poços com maior correlação foram Assis, Meridiano, Monte Aprazível, Penápolis, Pindorama e Valparaiso, pois tiveram valores de EVP maiores ou próximos a $70 \%$. Isso significa que os dados de precipitação que foram adquiridos em cada poço são satisfatórios nas analises. Mostrando o real ajuste da curva nível freático observado e a curva modelada. Outra característica que deve ser levada em consideração é o fator de o modelo ter se adequado a diferentes frequências de dados, e em cada um, ter realizado o melhor ajuste, sendo positivos para alguns poços e negativos para outros.

Mas mesmo com os valores de EVP relativamente baixos, todos os poços tiveram um RMSE $<1$, mostrando dessa forma a eficácia e qualidade do modelo gerado. Na figura 3 abaixo podemos verificar um exemplo de ajuste realizado para o poço de Assis.

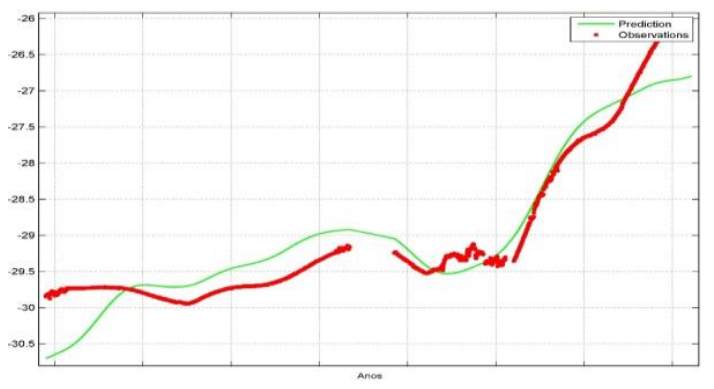

Figura 3. Comparação entre os dados observados (pontos) e calibrados (linhas) pelo modelo PIRFICT para o poço em Assis precipitação.

Conforme descrito acima, notase que o modelo (linha em verde) tenta se aproximar ao máximo dos níveis observados (linha em vermelho). Mas ele responde inexoravelmente de acordo com os dados de precipitação fornecidos. Caso esse dado não for de qualidade ou não estiver próximo ao poço, maior será a disparidade dos dados em relação ao real. No poço de Assis notamos que existe uma incongruência dos dados nos estágios iniciais, mas com adequação progressiva com o tempo. $\mathrm{O}$ modelo mostra uma aderência satisfatória, capaz de preencher lacunas de informação com alto grau de significância. $O$ modelo mostra-se eficiente eficiência para realizar predições estatísticas.
O modelo responde de forma satisfatória aos poços localizados nos três Estados, independente do sistema aquífero monitorado. Quanto maior a proximidade entre poço de monitoramento e a PCD, maior a aderência do modelo e maior a representatividade dos dados em geral.

$\mathrm{Na}$ modelagem do poço de União da Vitória o resultado foi um EVP < 50\%; mas mesmo assim o RMSE ficou < de $0.5 \mathrm{~m}$. Apesar da baixa EVP, a predição realizada foi considerada satisfatória. Mesmo com a correlação baixa dos parâmetros de precipitação e níveis freáticos, foi possível ter um RMSE menor do que o relatado em outros poços com características semelhantes. 


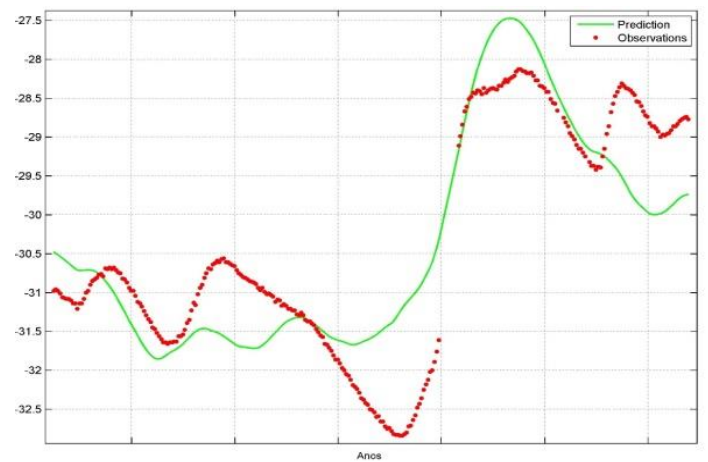

Figura 4. Comparação entre os dados observados (pontos) e calibrados (linhas) pelo modelo PIRFICT para o poço em Planaltina do Paraná precipitação.

No poço de Planaltina do Paraná (Figura 4), vemos que o modelo (linha em verde) foge um pouco dos parâmetros observados (linha em vermelho). Mesmo assim ele reproduz a resposta do aquífero, desde rebaixamento em períodos de estiagem à ascensão de nível com a retomada dos períodos chuvosos. Existem diversos fatores externos que podem acarretar em erros da modelagem, entre eles: (i) calibragem do equipamento, (ii) perda de dados de níveis no momento da coleta; (iii) excessiva distância dos dados de chuva em relação ao poço de monitoramento. Por outro lado, mesmo em poços com falhas de monitoramento de longo período, as respostas da modelagem são condizentes com a realidade.

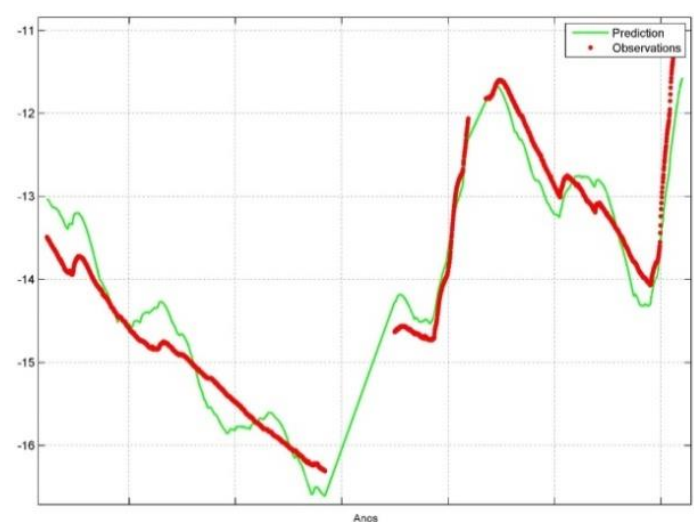

Figura 5. Comparação entre os dados observados (pontos) e calibrados (linhas) pelo modelo PIRFICT para o poço em Santa Rita do Rio Pardo precipitação.

Os poços de monitoramento modelados no Estado do Mato Grosso do Sul, todos integrados com PCD's forneceram correlações acima de $90 \%$ e RMSE $<0,5 \mathrm{~m}$. Na Figura 5 abaixo podemos verificar a modelagem realizada para o poço de Santa Rita do
Rio Pardo. Neste poço constata-se que o resultado modelado (linha em verde) se alinha de forma coincidente aos dados observados (linha em vermelho), preenchendo inclusive a série em períodos com falhas de monitoramento. 


\section{CONCLUSÃO}

O modelo PIRFICT mostrou-se muito adequado para a realização da modelagem das séries temporais dos níveis freáticos e as respectivas chuvas associadas. Possui robustez suficiente para manipular bancos de dados extensos compatíveis com a rede RIMAS. Demonstra muita sensibilidade ao parâmetro de entrada chuva e será

\section{REFERÊNCIAS BIBLIOGRÁFICAS}

BOX, G. E. P.; JENKINS, G. M. Time series analysis: forecasting and control.2.ed. San Francisco: HoldenDay, 1976. 575 p. Computer Systems (KBCS), 2002.

IRITANI, M. A.; ROSSINIPENTEADO, D.; EZAKI, S. ODA, G. H. Perigo de contaminação da água subterrânea na região de Indaiatuba a Capivari, estado de São Paulo, Brasil. Revista do Instituto Geológico, São Paulo, v.38, n.1, 1-16, 2017.

KIRCHHEIM, R., E., FRANZINI, A., S.; MATTIUZI, C., D., P., Avaliação crítica das disponibilidades hídricas subterrâneas em planos de bacia do Estado de São Paulo. Anais do Congresso Brasileiro de Águas Subterrâneas, 2018.

KNOTTERS, M.; BIERKENS, M. F. P. Physical basis of time series models for water table depths. Water Resources Research, v. 36, p. 181-188, 2000.

KWR W. Menyanthes. Disponível em: http://www.menyanthes.nl/\&gt;Acesso em: 20 fev. 2020.

LUIZ, T. B. P.; GAIARDO, G. F.; SILVA, J. L. S. Utilização de máquina de vetor para previsão de níveis de água subterrânea. Revista Águas tanto mais aderente quanto maior a representatividade espacial da mesma em relação ao poço de monitoramento. Trata-se de um modelo cuja aplicação pode ser usada para corrigir omissões nos registros históricos de níveis, assim como também para avaliar comportamentos freáticos esperados em períodos de grandes eventos climáticos.

Subterrâneas, v. 32 , n. 1, p. 25-34, 2018.

LUIZ, T. B. P.; SCHRÖDER, T.; SILVA, J. L. S. Previsão de Níveis Estáticos de Água Subterrânea em Poço de Monitoramento Utilizando Metodologia Estocástica. Anuário do Instituto de Geociências - UFRJ, v. 40, n. 2, p. 53-60, 2017.

MANZIONE, R. L. Águas Subterrâneas: Conceitos e aplicações sob uma visão interdisciplinar. Jundiaí: Paco Editorial, 2015. p. 185 - 210

MANZIONE, R. L.; MARCUZZO, F. FN; WENDLAND, E. Modelagem de níveis freáticos em área de recarga do Sistema Aquífero Guarani sob diferentes usos do solo. Águas Subterrâneas, 2010.

PINHEIRO, M. P. M. A.; MANZIONE, R. L. Modelagem do fluxo das águas subterrâneas em área de conservação no sudoeste paulista. Brazilian Journal of Biosystems Engineering, v. 13, n.3, p. 222-236, 2019

SANTOS, G. N; KIRCHHEIM, R. E.; LAZZAROTTO, E. M.; FRANZINI, A. S.; MOURÃO, M. A. A. Possíveis influências da Zona de Convergência do Atlântico Sul no comportamento freático e isotópico das águas 
subterrâneas do SAB e SAG (RIMAS) na região sudeste do Brasil. Anais do Congresso de Águas Subterrâneas, 2018.

TANKERSLEY, C. D.; GRAHAM, W. D. Development of an optimal control system for maintaining minimum groundwater levels. Water Resources Research, v. 30, p. 3171-3181, 1994.

TORRES, D. M.; NASCIMENTO, S. S.; SOUZA, J. F.; FREIRE, J.O. Tratamento de efluentes e produção de água de reúso para fins agrícolas. HOLOS, v.8, p. 1-15, 2019.

VAN GEER, F. C.; ZUUR, A. F. An extension of Box-Jenkins transfer/noise models for spatial interpolation of groundwater head series. Journal of Hydrology, v. 192, p. 65-80, 1997.

VON ASMUTH, J. R.; BIERKENS, M. F. P.; MAAS, C. Transfer function noise modelling in continuous time using predefined impulse response functions. Water Resources Research, v. 38; n.12, p. 23.1-23.12, 2002.

VON ASMUTH, J. R.; KNOTTERS, M. Characterising groundwater dynamics based on a system identification approach. Journal of Hydrology, v. 296, p. 118-34, 2004.

YI, M.; LEE, K. Transfer function-noise modelling of irregularly observed groundwater heads using precipitation data. Journal of Hydrology, v. 288, p. 272-287, 2003. 